\title{
Spacecraft Formation Reconfiguration using Impulsive Control Input
}

\author{
Jonghee Bae* and Youdan Kim** \\ Seoul National University, Seoul, South Korea
}

\begin{abstract}
This paper presents formation reconfiguration using impulsive control input for spacecraft formation flying. Spacecraft in a formation should change the formation size and/or geometry according to the mission requirements and space environment. To modify the formation radius and geometry with respect to the leader spacecraft, the follower spacecraft generates additional control inputs; the two impulsive control inputs are general control type of the spacecraft system. For the impulsive control input, Lambert's problem is modified to construct the transfer orbit in relative motion, given two position vectors at the initial and final time. Moreover, the numerical simulation results show the transfer trajectories to resize the formation radius in the radial/along-track plane formation and in the along-track/cross-track plane formation. In addition, the maneuver characteristics are described by comparing the differential orbital elements between the reference orbit and transfer orbit in the radial/along-track plane formation and along-track/cross-track plane formation.
\end{abstract}

Key words: Spacecraft Formation Flying, Formation Reconfiguration, Impulsive Control Input, Lambert's Problem

\section{Introduction}

Spacecraft formation flying has been widely investigated due to increasing interests in the design of clusters and their application. Multiple spacecraft in formation have many advantages in terms of flexibility, reliability, financial benefits, and high image resolution compared to a single large spacecraft. Autonomous formation flying has been developed for future space missions in many countries. National Aeronautics and Space Administration (NASA) provided the Earth-Observing 1 (EO-1), StarLight (ST) series, Terrestrial Planet Finder (TPF), Micro-Arcsecond X-ray Imaging Mission (MAXIM), and Magnetospheric Multiscale (MMS) mission using multiple spacecraft [1-4]. Moreover, European Space Agency (ESA) developed the Cluster II mission, the Dawin, the $\mathrm{X}$-ray Evolving Universe Spectroscopy (XEUS) missions, and PROBA-3 consisting of multiple spacecraft for various missions $[5,6]$. The Laser Interferometer Space Antenna (LISA) was designed to develop a space-based gravitational wave detector by NASA and ESA. During the spacecraft operation, formation reconfiguration may be needed to change the formation radius and/or geometry according to the mission requirements. For formation reconfiguration, the follower spacecraft should modify the orbit with respect to the leader spacecraft by additional control inputs.

Lots of works on the maneuver problem have been solved in aninertialframe. Thegoals of the traditionalmaneuverproblem are to change the orbit size or shape of a single spacecraft with respect to a planet considering mission requirements, and to minimize the control effort during the maneuver. For spacecraft formation, on the other hand, the formation can be reconfigured by increasing or decreasing the formation radius and/or changing the formation geometry through maneuver in relative motion with respect to the leader spacecraft. This transfer between two orbits can be generally performed by impulsive control input. The traditional maneuver problem for spacecraft orbit transfer has been solved by two impulsive control inputs. The Hohmann transfer is well known as twoburn minimum fuel transfer in circular coplanar orbits [7, 8]. For the general two-impulse maneuver problem between two fixed position vectors with the specified flight time, the transfer orbit can be determined by solving Lambert's
This is an Open Access article distributed under the terms of the Creative Commons Attribution Non-Commercial License (http://creativecommons.org/licenses/by$\mathrm{nc} / 3.0 /$ which permits unrestricted non-commercial use, distribution, and reproduction in any medium, provided the original work is properly cited. (c) $\quad$ Postdoctoral Researcher
* * Professor, Corresponding author: ydkim@snu.ac.kr 
problem [8-10], and therefore the required velocity vectors of the resulting transfer orbit can be calculated at the initial and final time. Many researchers utilized Lambert's problem to obtain the transfer trajectory between two orbits with respect to a planet [11-13]. Won considered minimum-fuel and minimum-time orbit transfer problem in two coplanar elliptic orbits [11]. Prussing considered the minimumfuel impulsive spacecraft trajectory through the multiplerevolution [12], and Shen and Tsiotras studied the optimal fixed-time, two-impulse rendezvous problem between two satellites in coplanar circular orbit using multiple-revolution Lambert's problem [13].

Although the traditional maneuver problem has been widely studied, the formation reconfiguration problem is different from that because the formation of multiple spacecraft is expressed by the relative motion dynamics with respect to the leader spacecraft, not a planet. Vaddi et al. studied the formation reconfiguration problem using impulsive control based on Gauss's variational equation [14], and Ketema considered the optimal transfer problem using two impulsive control inputs considering the relative motion of the spacecraft formation [15]. However, the transfer method for the follower spacecraft from one orbit to another with respect to the leader spacecraft in the local frame, where the initial and final positions, velocities, transfer time, and orbital elements of the leader spacecraft are specified, has not been sufficiently investigated. In this study, the traditional spacecraft maneuver method, Lambert's problem, is modified to design the transfer orbit trajectory of the follower spacecraft, where two relative position vectors and the flight time in the local frame are given. Furthermore, the characteristics of the formation reconfiguration in two formation geometries, radial/along-track plane formation and along-track/cross-track plane formation, are analyzed in terms of the orbital elements. The numerical simulation results show that the transfer trajectories are designed to resize the formation radius in two formation geometries.

This paper is organized as follows. In Section 2, the relative motion dynamics are described. Spacecraft formation reconfiguration using impulsive control input is derived in Section 3 , and the numerical simulation results and analysis to verify the formation reconfiguration are shown in Section 4. Finally, conclusion is presented in Section 5.

\section{Spacecraft Relative Motion Dynamics}

The inertial coordinate system and a rotating local coordinate system are defined for spacecraft formation flying. As shown in Fig. 1, the inertial coordinate system $\{X$,
$Y, Z\}$ is attached to the center of the Earth, and a rotating local-vertical-local-horizontal (LVLH) Cartesian frame $\{x$, $y, z\}$ is attached to the center of the leader spacecraft. The $x$-axis points in the radial direction, the $y$-axis points in the along-track direction, and the z-axis is perpendicular to the orbital plane and points in the direction of the angular momentum vector. $\vec{R}=\left\{\begin{array}{lll}R & 0 & 0\end{array}\right\} \in \mathfrak{R}^{3}$ denotes the position vector of the leader spacecraft and $\vec{\rho}=\left\{\begin{array}{lll}x & y & z\end{array}\right\} \in \mathfrak{R}^{3}$ is the relative position vector of the follower spacecraft with respect to the leader spacecraft in the LVLH frame. Then, the position of each follower spacecraft within a formation is given by

$$
\vec{R}_{f}=\vec{R}+\vec{\rho}
$$

The nonlinear equation of the relative motion for elliptic reference orbits can be represented as

$$
\ddot{\vec{\rho}}+2 \vec{\omega} \times \dot{\vec{\rho}}+\dot{\vec{\omega}} \times \vec{\rho}+\vec{\omega} \times \vec{\omega} \times \vec{\rho}=\vec{f}_{f}-\vec{f}
$$

where $\vec{\omega} \in \mathfrak{R}^{3}$ is the angular velocity, and $\vec{f} \in \mathfrak{R}^{3}$ and $\vec{f}_{f} \in \mathfrak{R}^{3}$ are external forces acting on the leader and follower spacecraft, respectively. When the external forces only act on a central gravitational field, the nonlinear relative dynamics for elliptic reference orbits can be represented as follows:

$$
\begin{aligned}
& \ddot{x}=2 \dot{\theta} \dot{y}+\ddot{\theta} y+\dot{\theta}^{2} x-\frac{\mu(R+x)}{\left\{(R+x)^{2}+y^{2}+z^{2}\right\}^{3 / 2}}+\frac{\mu}{R^{2}}+u_{x} \\
& \ddot{y}=-2 \dot{\theta} \dot{x}+\ddot{\theta} x+\dot{\theta}^{2} y-\frac{\mu y}{\left\{(R+x)^{2}+y^{2}+z^{2}\right\}^{3 / 2}}+u_{y} \\
& \ddot{z}=-\frac{\mu z}{\left\{(R+x)^{2}+y^{2}+z^{2}\right\}^{3 / 2}}+u_{z}
\end{aligned}
$$

where $\mu$ is the gravitational coefficient, $\theta$ is the argument of latitude of the leader spacecraft, $\dot{\theta}$ is the orbital angular velocity, and $u_{x}, u_{y}$, and $u_{z}$ are the control inputs in the

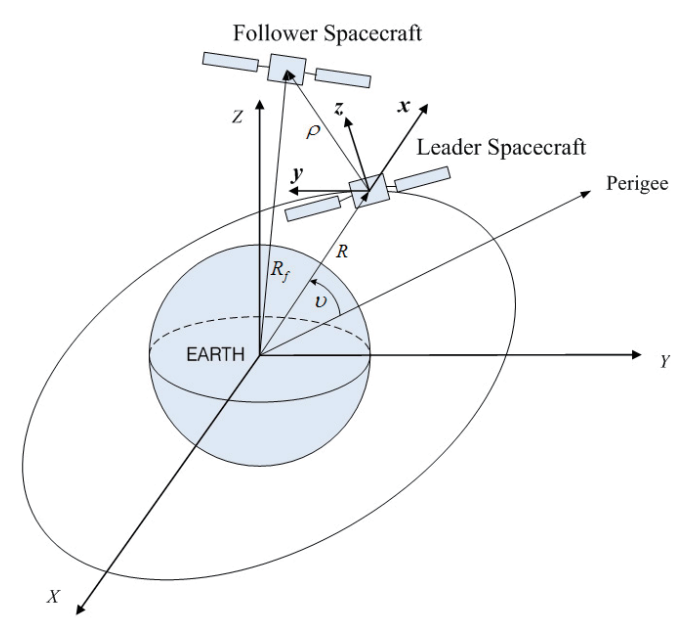

Fig. 1. Local Vertical Local Horizontal frame 
radial, along-track, and cross-track direction, respectively. In orbital mechanics, the radius and angular velocity of the leader spacecraft are given as [16]

$$
R=\frac{a\left(1-e^{2}\right)}{1+e \cos v}, \quad \dot{\theta}=\frac{n(1+e \cos v)^{2}}{\left(1-e^{2}\right)^{3 / 2}}
$$

where $a$ is the semi-major axis, e is the eccentricity, $v$ is the true anomaly, and $n=\sqrt{\mu / a^{3}}$ is the natural frequency of the reference orbit.

Equation (3) can be expressed using the true anomaly $v$ as a free variable, because $v$ of the reference orbit monotonically increases and describes the angular velocity. The following transformation is considered:

$$
\begin{aligned}
& \text { ( ) }=()^{\prime} \dot{\theta} \\
& (\quad)=()^{\prime \prime} \dot{\theta}^{2}+\dot{\theta} \dot{\theta}^{\prime}()^{\prime} \\
& \dot{\theta}=\dot{v}=\frac{\sqrt{\mu p}}{R^{2}}=\sqrt{\frac{\mu}{p^{3}}}(1+e \cos v)^{2}
\end{aligned}
$$

where ( )' and ( )" denote the first and second derivatives with respect to $v$. With the transformation of Eq. (5) and the assumption that $|\vec{\rho}|<<|\vec{R}|$, the relative motion equation can be expressed as follows:

$$
\begin{aligned}
x^{\prime \prime} & =\frac{2 e \sin v}{1+e \cos v} x^{\prime}+2 y^{\prime}+\frac{3+e \cos v}{1+e \cos v} x-\frac{2 e \sin v}{1+e \cos v} y+\frac{\left(1-e^{2}\right)^{3}}{n^{2}(1+e \cos v)^{4}} u_{x} \\
y^{\prime \prime} & =-2 x^{\prime}+\frac{2 e \sin v}{1+e \cos v} y^{\prime}+\frac{2 e \sin v}{1+e \cos v} x+\frac{e \sin v}{1+e \cos v} y+\frac{\left(1-e^{2}\right)^{3}}{n^{2}(1+e \cos v)^{4}} u_{y}(7) \\
z^{\prime \prime} & =\frac{2 e \sin v}{1+e \cos v} z^{\prime}-\frac{1}{1+e \cos v} z+\frac{\left(1-e^{2}\right)^{3}}{n^{2}(1+e \cos v)^{4}} u_{z}
\end{aligned}
$$

Using Eq. (7), the maneuver problem for the formation reconfiguration is solved and the initial and final conditions are selected in the true anomaly domain to meet the periodicity condition as presented in Ref. [17].

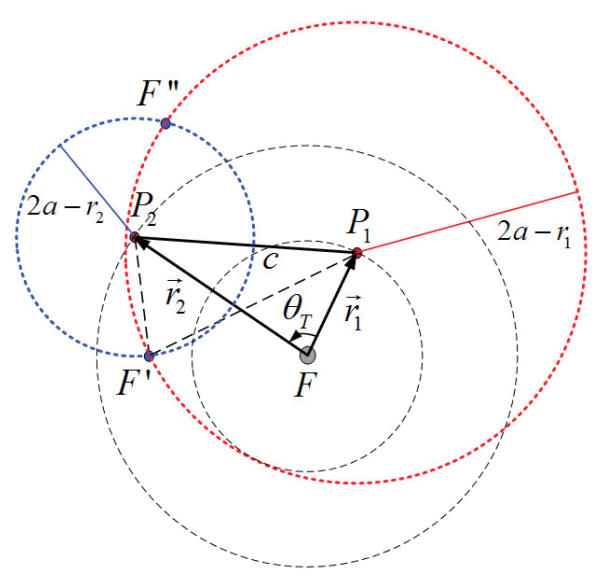

Fig. 2. Geometry for Lambert's proble

\section{Formation Reconfiguration using Impul sive Control Input}

\subsection{Lambert's Problem}

Lambert's theorem states that the flight time from one point to another depends on the semi-major axis of transfer orbit, the distance of the initial and final points of the arc from the center of force, and the length of the chord joining the points. The chord length, $c$, between two position vectors $\vec{r}_{1}$ and $\vec{r}_{2}$, as shown in Fig. 2, is defined by the cosine law as follows:

$$
c=\sqrt{r_{1}^{2}+r_{2}^{2}-2 r_{1} r_{2} \cos \left(\theta_{T}\right)}
$$

In Fig. 2, $F$ is the primary focus which is the location of the Earth, $\vec{r}_{1}$ and $\vec{r}_{2}$ are the position vectors at the initial time $t_{1}$ and the final time $t_{2}$, and $\theta_{T}$ is the transfer angle between two position vectors defined by

$$
\cos \left(\theta_{T}\right)=\frac{\vec{r}_{1} \cdot \vec{r}_{2}}{r_{1} r_{2}}
$$

The direction of flight can be defined by the transfer angle; the spacecraft moves along the short way when $\theta_{T} \leq \pi$, and it moves along the long way when $\theta_{T}>\pi$.

To determine the transfer orbit between two position vectors, two foci and semi-major axis should be found. From the definition of the ellipse, the location of the vacant focus and semi-major axis of the transfer orbit can be determined. As shown in Fig. 2, two circles can be drawn having $2 a-r_{1}$ and $2 a-r_{2}$ as radii and points $P_{1}$ and $P_{2}$ as centers, respectively, since the sum of the distance from the foci to any point on the ellipse equals twice the semi-major axis as $2 a=r_{1}+(2 a$ $\left.r_{1}\right)=r_{2}+\left(2 a-r_{2}\right)$. As a result, two intersected points, $F^{\prime}$ and $F^{\prime \prime}$, are determined as shown in Fig. 2, which are the candidate

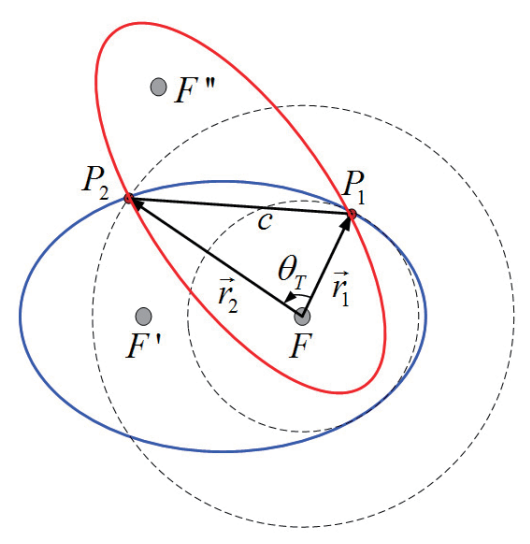


locations of other focus of the transfer orbit. Using two vacant foci, two elliptic orbits can be obtained as shown in Fig. 2. Note from Fig. 2 that two ellipses have different eccentricities; the ellipse with vacant focus $F^{\prime}$ has the smaller eccentricity, and the ellipse with $F^{\prime \prime}$ has the larger eccentricity. The location of vacant focus depends on the semi-major axis of the transfer orbit, and therefore the semi-major axis should be determined carefully through iterative calculation for the minimum energy.

When the transfer orbit is determined, the velocity vectors of the transfer orbit at the initial and final time, $\vec{v}_{t, 1}$ and $\vec{v}_{t, 2}$ , as shown in Fig. 3, can be calculated by Kepler's equation. Consequently, the velocity changes $\Delta v_{1}$ and $\Delta v_{2}$ at $t_{1}$ and $t_{2}$ can be obtained as

$$
\begin{aligned}
& \Delta v_{1}=\vec{v}_{t, 1}-\vec{v}_{1} \\
& \Delta v_{2}=\vec{v}_{2}-\vec{v}_{t, 2}
\end{aligned}
$$

where $\vec{v}_{1}$ and $\vec{v}_{2}$ are velocity vectors of the spacecraft around a planet at the initial and final time. Figure 3 shows the velocity changes using two impulse inputs. The total velocity change can defined as follows:

$$
\Delta v=\left|\Delta v_{1}\right|+\left|\Delta v_{2}\right|
$$

\subsection{Modified Lambert's Problem for Follower Space- craft}

The transfer orbit for the follower spacecraft connecting two relative position vectors can be solved modifying Lambert's problem, when the orbital elements of the leader spacecraft and the flight time are known. The geometry of the transfer orbit for the follower spacecraft is illustrated in Fig. 4.

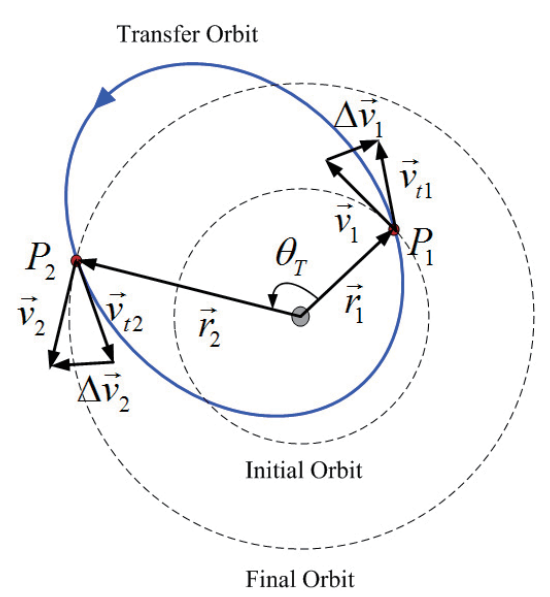

Fig. 3. Orbital transfer using two impulsive control inputs
The relative position vectors of the follower spacecraft with respect to the leader spacecraft, $\vec{\rho}_{1} \in \mathfrak{R}^{3}$ and $\vec{\rho}_{2} \in \mathfrak{R}^{3}$ at the initial time $t_{1}$ and the final time $t_{2}$ in the LVLH frame, respectively, can be expressed as follows:

$$
\begin{aligned}
& \vec{\rho}_{1}=\left[\begin{array}{lll}
x_{1} & y_{1} & z_{1}
\end{array}\right]^{T} \\
& \vec{\rho}_{2}=\left[\begin{array}{lll}
x_{2} & y_{2} & z_{2}
\end{array}\right]^{T}
\end{aligned}
$$

The position vectors of the follower spacecraft with respect to the Earth at $t_{1}$ and $t_{2}$ in the ECI (Earth Centered Inertial) frame can be written as

$$
\begin{aligned}
& \vec{R}_{1}=\vec{r}_{1}+\vec{\rho}_{1} \\
& \vec{R}_{2}=\vec{r}_{2}+\vec{\rho}_{2}
\end{aligned}
$$

where $\vec{r}_{1} \in \mathfrak{R}^{3}$ and $\vec{r}_{2} \in \mathfrak{R}^{3}$ are the position vectors of the leader spacecraft in the ECI frame at $t_{1}$ and $t_{2}$, respectively.

Let us define the velocity vectors of the follower spacecraft at $t_{1}$ and $t_{2}$ in the ECI frame as $\vec{V}_{1} \in \mathfrak{R}^{3}$ and $\vec{V}_{2} \in \mathfrak{R}^{3}$ , respectively. Given two position vectors, $\vec{R}_{1} \in \mathfrak{R}^{3}$ and $\vec{R}_{2} \in \mathfrak{R}^{3}$, the transfer orbit for the follower spacecraft can be determined through Lambert's problem as explained in the previous section. The transfer angle can be defined by

$$
\cos \left(\theta_{T}\right)=\frac{\vec{R}_{1} \cdot \vec{R}_{2}}{R_{1} R_{2}}
$$

The chord length is determined as

$$
c=\sqrt{R_{1}^{2}+R_{2}^{2}-2 R_{1} R_{2} \cos \left(\theta_{T}\right)}
$$

Using $\theta_{T}$ and $c$, Lambert's problem can provide the velocities of the transfer orbit, $\vec{V}_{1, t} \in \mathfrak{R}^{3}$ and $\vec{V}_{2, t} \in \mathfrak{R}^{3}$, as shown in Fig. 4. The transfer time for the elliptic orbit is defined from Kepler's equation as follows [7, 8]:

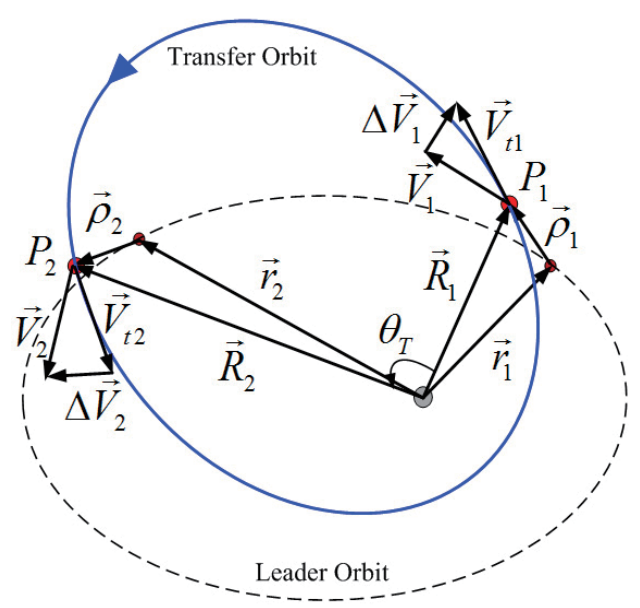

Fig. 4. Orbit transfer for follower spacecraft 


$$
\Delta t=\sqrt{\frac{a^{3}}{\mu}}\left[2 \pi k+\left(E_{2}-e \sin E_{2}\right)-\left(E_{1}-e \sin E_{1}\right)\right]
$$

where $\triangle t=t_{2}-t_{1}$, and $E_{1}$ and $E_{2}$ are eccentric anomalies at $t_{1}$ and $t_{2}$. In this study, multiple revolutions are not considered, and then $2 \pi k$ term in Eq. (18) can be ignored. Equation (18) can be rewritten as

$$
\sqrt{\mu} \Delta t=\sqrt{a^{3}}\left[\Delta E+e\left(\sin E_{1}-\sin E_{2}\right)\right]
$$

where $\triangle E=E_{2}-E_{1}$. To solve Kepler's equation, the universal variable, $\chi$, is adopted in this study, which relates energy and angular momentum [8]. The universal variable, $\chi$ is defined as follows:

$$
\chi=\sqrt{a} \Delta E
$$

In addition, parameters $s_{2}$ and $s_{3}$ are defined as [8]

$$
s_{2}=\frac{1-\cos \Delta E}{\Delta E^{2}}, \quad s_{3}=\frac{\Delta E-\sin \Delta E}{\Delta E^{3}}
$$

Using Eqs. (20) and (21), Eq. (19) can be expressed as

$$
\sqrt{\mu} \Delta t=\chi^{3} s_{3}+\sqrt{a^{3}} \sin \Delta E+\sqrt{a^{3}} e\left(\sin E_{1}-\sin E_{2}\right)
$$

With trigonometric identity, $\sin \left(a_{1}-a_{2}\right)=\sin a_{1} \cos a_{2}$ $\cos a_{1} \sin a_{2}$, Eq. (22) can be rewritten as

$$
\sqrt{\mu} \Delta t=\chi^{3} s_{3}+\sqrt{a^{3}}\left(\sin E_{1}\left(e-\cos E_{2}\right)-\sin E_{2}\left(e-\cos E_{1}\right)\right)
$$

The relation between eccentric anomaly $E$ and true anomaly $v$ is defined by $[7,8]$

$$
\cos v=\frac{e-\cos E}{e \cos E-1}
$$

After some manipulation with Eq. (24), Eq. (23) can be expressed as follows:

$$
\sqrt{\mu} \Delta t=\chi^{3} s_{3}+\sqrt{a^{3}}\left(\sin v_{2} \cos v_{1}-\sin v_{1} \cos v_{2}\right)\left[\frac{\left(1-e \cos E_{1}\right)\left(1-e \cos E_{2}\right)}{\sqrt{1-e^{2}}}\right]
$$

With the trigonometric identity, Eq. (25) is presented as

$$
\sqrt{\mu} \Delta t=\chi^{3} s_{3}+\sqrt{a^{3}} \sin \theta_{T} \frac{\left(1-e \cos E_{1}\right)\left(1-e \cos E_{2}\right)}{\sqrt{1-e^{2}}}
$$

The radius is defined as $r=a(1-e \cos E)$ in orbital mechanics, and then Eq. (26) can be rewritten as

$$
\sqrt{\mu} \Delta t=\chi^{3} s_{3}+A \sqrt{Y}
$$

where

$$
A=\frac{\sqrt{R_{1} R_{2}} \sin \theta_{T}}{\sqrt{1-\cos \theta_{T}}}, \quad Y=\frac{\sqrt{R_{1} R_{2}\left(1-\cos \theta_{T}\right)}}{\sqrt{a\left(1-e^{2}\right)}}
$$

The resulting $A$ and $Y$ give the velocity vectors of the transfer orbit at $t_{1}$ and $t_{2}$ as follows:

$$
\begin{aligned}
& \vec{V}_{t, 1}=\frac{\vec{R}_{2}-f \vec{R}_{1}}{g} \\
& \vec{V}_{t, 2}=\frac{\dot{g} \vec{R}_{2}-\vec{R}_{1}}{g}
\end{aligned}
$$

where

$$
\begin{array}{ll}
f=1-\frac{Y}{R_{1}} & \\
g=A \sqrt{\frac{Y}{\mu}}, \quad \dot{g}=1-\frac{Y}{R_{2}}
\end{array}
$$

Finally, the velocity changes $\triangle V_{1}$ and $\triangle V_{2}$ for the follower spacecraft at $t_{1}$ and $t_{2}$ can be obtained as follows:

$$
\begin{aligned}
& \Delta V_{1}=\vec{V}_{t, 1}-\vec{V}_{1} \\
& \Delta V_{2}=\vec{V}_{2}-\vec{V}_{t, 2}
\end{aligned}
$$

Consequently, the total velocity change can be calculated as

$$
\Delta V=\left|\Delta V_{1}\right|+\left|\Delta V_{2}\right|
$$

Note that $\Delta \vec{V}_{t, 1}$ and $\Delta \vec{V}_{t, 2}$ are velocities with respect to the Earth and expressed in the ECI frame. Thus, the relative velocity vectors of the follower spacecraft with respect to the leader spacecraft can be expressed as follows:

$$
\begin{aligned}
& \dot{\vec{\rho}}_{1}=\vec{V}_{t, 1}-\vec{v}_{1}-\omega \times \vec{\rho}_{1} \\
& \dot{\vec{\rho}}_{2}=\vec{V}_{t, 2}-\vec{v}_{2}-\omega \times \vec{\rho}_{2}
\end{aligned}
$$

where $\vec{v}_{1} \in \mathfrak{R}^{3}$ and $\vec{v}_{2} \in \mathfrak{R}^{3}$ are velocity vectors of the leader spacecraft at $t_{1}$ and $t_{2}$, respectively, and $\omega$ is the angular velocity vector of the leader spacecraft as defined by

$$
\omega=\dot{v} \vec{k}, \quad \vec{k}=\frac{\vec{r}_{1} \times \vec{r}_{2}}{\left\|\vec{r}_{1} \times \vec{r}_{2}\right\|}
$$

Finally, the relative velocity vectors in the LVLH frame and the orbital elements of the transfer orbit can be found.

\section{Numerical Simulation and Analysis}

In this section, numerical simulations are performed for the maneuver problem of formation reconfiguration. Using impulsive control input, two maneuvering cases are considered to verify the formation reconfiguration in relative motion: change of the formation radius, $r_{d}$ (i) in the radial/ 
along-track plane formation (RAPF) and (ii) in the alongtrack/cross-track plane formation (ACPF).

\subsection{Radial/Along-Track Plane Formation}

In this section, the maneuver for formation reconfiguration in the RAPF is performed using impulsive control input. The orbital elements of the leader spacecraft are summarized in Table 1. To change the formation radius in the RAPF, we consider a situation that the follower spacecraft moves from the initial formation radius of $5,000 \mathrm{~m}$ to the final formation radius of $10,000 \mathrm{~m}$. The initial and final conditions are selected to satisfy the periodicity and zero offset condition according to the formation geometry as presented in Ref. [17]. The initial and final conditions of the follower spacecraft in the LVLH frame are summarized in Table 2. The flight time is obtained as 5166.4 seconds given the initial and final conditions as described in Table 2.

Table 1. Orbital elements of leader spacecraft

\begin{tabular}{cc}
\hline Parameter & Value \\
\hline$a(\mathrm{~m})$ & $1.000 \times 10^{7}$ \\
$e$ & 0.1 \\
$i$ & 66.01 \\
$\Omega(\operatorname{deg})$ & 277 \\
$\omega(\operatorname{deg})$ & 45 \\
\hline
\end{tabular}

Figures 5-7 show the simulation results for maneuvering in the RAPF. Figure 5 shows the trajectories of the leader and follower spacecraft in the ECI frame; the normal line denotes the trajectory of the leader spacecraft and the thick line shows the transfer trajectory of the follower spacecraft. As shown in Fig. 5, it is difficult to understand the relative motion of the follower spacecraft with respect to the leader spacecraft in the ECI frame. Instead, Fig. 6 shows the transfer trajectory of the follower spacecraft in the LVLH frame. As shown in Fig. 6, the follower spacecraft moves from the initial position to the final position on the $x$ - $y$ plane in the LVLH frame. Figure 7 describes the relative velocity history of the follower spacecraft during the maneuver. In Fig. 7, the triangle denotes specified relative velocities at the initial and final time in the LVLH frame given in Table 2. As a result, the difference between the resulting velocity from the modified Lambert's problem and the specified velocity is equal to the required velocity change for the orbit transfer. Table 3 summarizes the relative velocities of the follower spacecraft and the velocity change, $\triangle V$, for the reconfiguration maneuver in the RAPF.

\subsection{Along-Track/Cross-Track Plane Formation}

In this section, the maneuver for formation reconfiguration in the ACPF is performed using impulsive control input. The orbital elements of the leader spacecraft are same as previous section. To change the formation radius in the ACPF, we consider a situation in which the follower spacecraft moves

Table 2. Initial and final condition (RAPF)

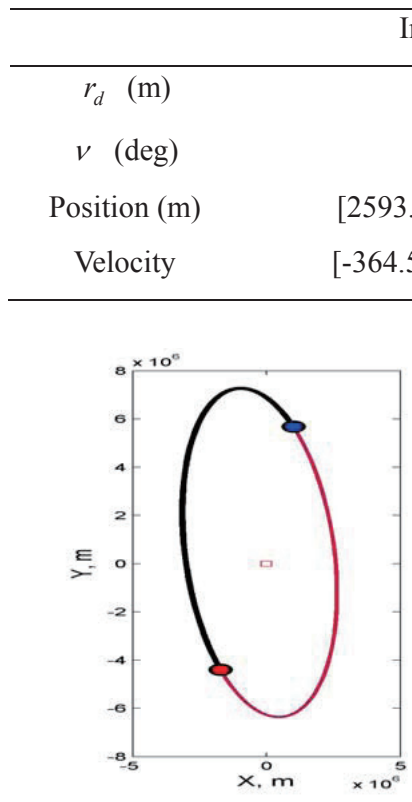

Initial condition Final condition

5,000

10,000

98

262

$[-5187.1185,-1468.2929,0]$

$[-729.0020,10521.6894,0]$
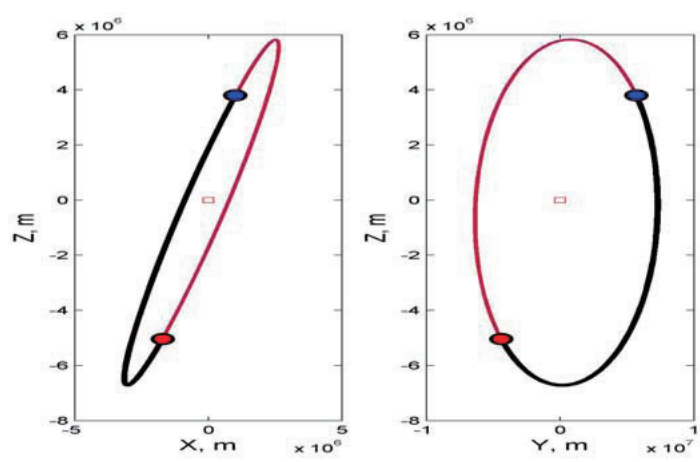

Fig. 5. Trajectory of two spacecraft in ECI frame (RAPF) 

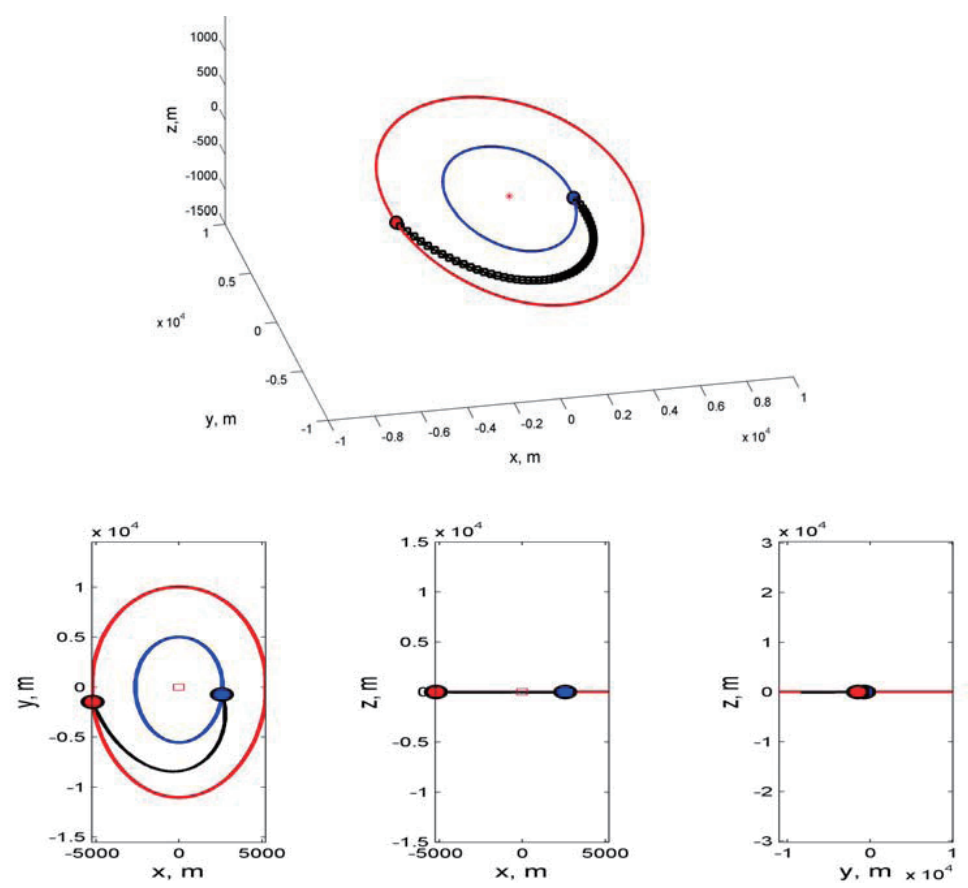

Fig. 6. Trajectory of follower spacecraft in LVLH frame (RAPF)
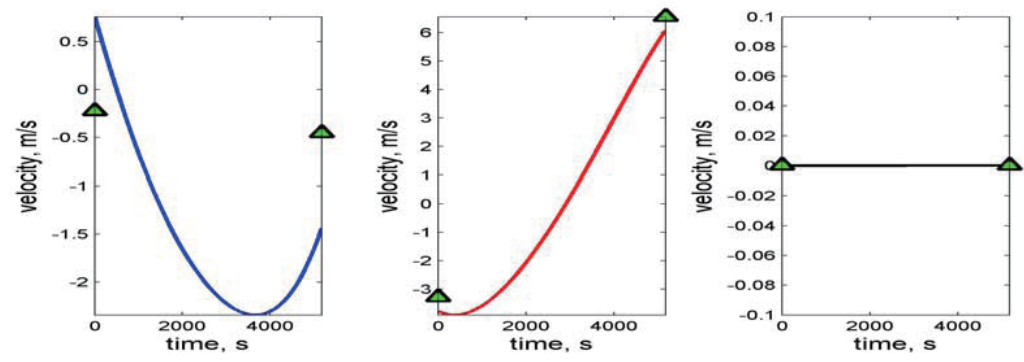

Fig. 7. Velocity history of follower spacecraft (RAPF)

Table 3. Solution of modified Lambert's problem (RAPF)

\begin{tabular}{ccc}
\hline & Initial value & Final value \\
\hline velocity $(\mathrm{m} / \mathrm{s})$ & {$[-0.0398,0,0]$} & {$[-0.0797,0,0]$} \\
$V_{t}(\mathrm{~m} / \mathrm{s})$ & {$[0.1328,-0.6606,0]$} & {$[-0.2523,1.0638,0]$} \\
$\Delta V(\mathrm{~m} / \mathrm{s})$ & {$[0.1726,-0.6606,0]$} & {$[0.1726,-1.0638,0]$} \\
Total $\Delta V$ & {$[0.3453,1.7244,0]$} & Norm: 1.7587
\end{tabular}

Table 4. Initial and final condition (ACPF)

\begin{tabular}{ccc}
\hline & Initial condition & Final condition \\
\hline$r_{d}(\mathrm{~m})$ & 5,000 & 10,000 \\
$v(\mathrm{deg})$ & 98 & 262 \\
Position $(\mathrm{m})$ & {$[2593.5592,-734.1464,5260.3281]$} & {$[-5187.1185,-1468.2929,-10520.6561]$} \\
Velocity & {$[-364.5010,0,-211.0254]$} & {$[-729.0020,0,-422.0507]$} \\
\hline
\end{tabular}


from the initial formation radius of $5,000 \mathrm{~m}$ to the final formation radius of $10,000 \mathrm{~m}$. The initial and final conditions of the follower spacecraft in the LVLH frame are given by formation geometry in Table 4 as presented in Ref. [17].

Figures 8-9 show the simulation results for formation reconfiguration using impulsive control input in the
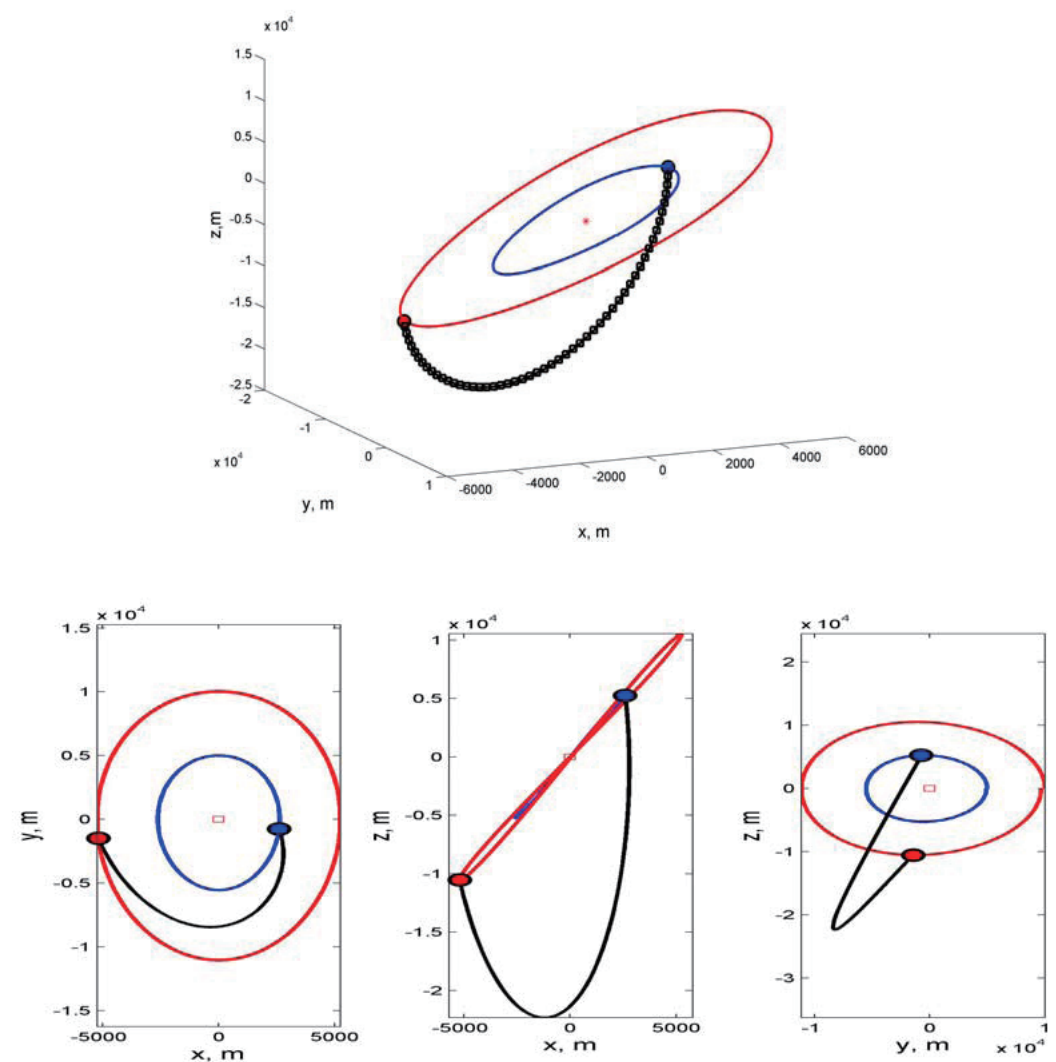

Fig. 8. Trajectory of follower spacecraft in LVLH frame (ACPF)
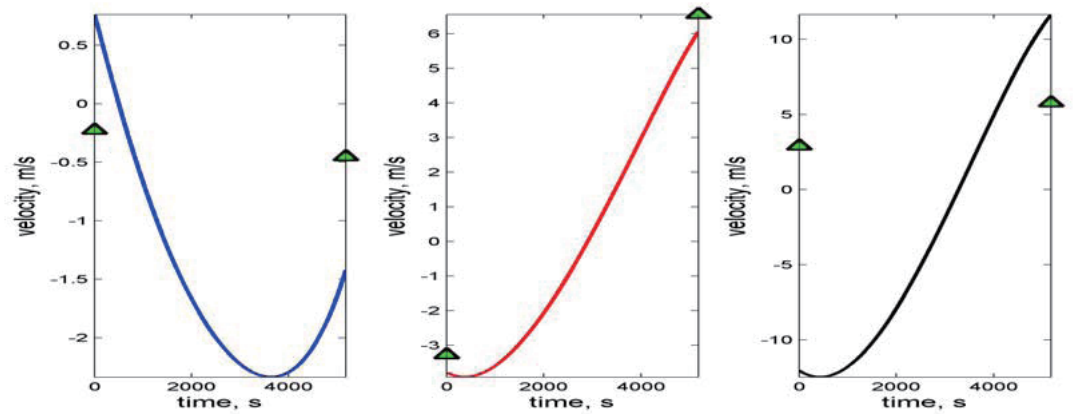

Fig. 9. Velocity history of follower spacecraft (ACPF)

Table 5. Solution of modified Lambert's problem (ACPF)

\begin{tabular}{ccc}
\hline & Initial value & Final value \\
\hline velocity $(\mathrm{m} / \mathrm{s})$ & {$[-0.2272,0,-0.1315]$} & {$[-0.4544,0,-0.2630]$} \\
$V_{t}(\mathrm{~m} / \mathrm{s})$ & {$[0.7622,-3.7804,-12.0337]$} & {$[-1.4248,6.0572,11.6496]$} \\
$\Delta V \quad(\mathrm{~m} / \mathrm{s})$ & {$[0.9894,-3.7804,-11.9022]$} & {$[0.9705,-6.0572,-11.9126]$} \\
Total $\Delta V$ & {$[1.9599,9.8376,23.8148]$} & Norm: 25.8412
\end{tabular}


ACPF. Figure 8 shows the transfer trajectory of the follower spacecraft with respect to the leader spacecraft in the LVLH frame. Figure 9 shows the velocity history of the follower spacecraft during the maneuver. In Fig. 9, the triangle denotes specified velocities at the initial and final time in the LVLH frame given in Table 4. Therefore, the required velocity change can be obtained by computing the difference between the resulting velocity from the modified Lambert's problem and the specified velocity. Table 5 summarizes the velocities of the follower spacecraft and $\triangle V$ for the maneuver in the ACPF.

\subsection{Analysis}

In this section, the simulation results for the formation reconfiguration using impulsive control input in the RAPF and ACPF are analyzed. The simulation results are investigated in terms of the difference of the orbital elements between the transfer orbit and reference orbit with respect to the formation geometry.

As described in Sections 4.1 and 4.2, the transfer orbits are generated where the initial and final positions are arbitrarily chosen in the RAPF and ACPF. By comparing with the orbital elements of the leader spacecraft in Table 1, it is seen that the transfer orbits of two formation reconfigurations have almost same orbital elements, as described in Fig. 5 ; however, some orbital elements are different. Table 6 summarizes the differences of the orbital elements: (i) between the reference orbit and the transfer orbit in the RAPF as described in Sections 4.1, and (ii) between the reference orbit and the transfer orbit in the ACPF in Section 4.2. These results show the maneuver characteristics of relative motion in the RAPF and ACPF. As shown in Table 6 , the difference of the semi-major axis for the ACPF case is much larger than that for the RAPF case. This difference leads to the total velocity change $\triangle V$ for the formation reconfiguration; it can be seen in Tables 3 and 5 that $\Delta V$ for resizing the formation radius in the ACPF is increased compared with $\triangle V$ in the RAPF as $\triangle a$ in the ACPF becomes large. Furthermore, as represented in Table 6, the transfer between two orbits in RAPF produces a difference in orbital elements including the semi-major axis $a$, eccentricity $e$, and augment of perigee $\omega$, which means that orbit transfer in the RAPF belongs to the coplanar maneuver. On the other hand, in the ACPF, not only $a, e$, and $\omega$, but also inclination $\mathrm{i}$ and longitude of the ascending node $\Omega$ are changed during the maneuver, as shown in Table 6. It means that the orbit transfer in the ACPF is related to the combined maneuver of coplanar and non-coplanar maneuvers.

\section{Conclusion}

A formation reconfiguration using impulsive control input is developed for spacecraft formation flying. To transfer between two position vectors at initial and final time, two impulsive control inputs are generated through modification of the classical Lambert's problem. The modified method provides the transfer orbit in relative motion for spacecraft formation flying, and therefore the follower spacecraft can change the formation radius and/or geometry with respect to the leader spacecraft. Numerical simulations are performed to solve the maneuver problem for formation reconfiguration in the RAPF and ACPF. These results show the maneuver characteristics of the transfer orbit with respect to the formation type. Through these results, it can be concluded that the transfer orbit for resizing formation radius in the RAPF is related to the coplanar maneuver, and transfer orbit in the ACPF belongs to the combined maneuver of coplanar and non-coplanar maneuvers.

\section{Acknowledgement}

This work has been supported by National GNSS Research Center program of Defense Acquisition Program Administration and Agency for Defense Development.

\begin{tabular}{ccc}
\hline & RAPF & ACPF \\
\hline$\Delta a(\mathrm{~m})$ & 1.2256 & 11.5291 \\
$\Delta e$ & 0.0002 & 0.0003 \\
$\Delta i(\mathrm{deg})$ & 0 & 0.0015 \\
$\Delta \Omega(\mathrm{deg})$ & 0 & 0.0013 \\
$\Delta \omega(\mathrm{deg})$ & 0.0013 & 0.0111 \\
\hline
\end{tabular}




\section{References}

[1] Folta, D., and Hawkins, A., "Results of NASA's First Autonomous Formation Flying Experiment: Earth Observing-1 (EO-1)", AIAA/AAS Astrodynamics Specialist Conference, Monterey, CA, 2002.

[2] Beichman, C. A., Woolf, N. J., and Lindensmith, C. A., The Terrestrial Planet Finder (TPF): A NASA Origins Program to Search for Habitable Planets, JPL Publication, Pasadena, CA, 1999.

[3] Folta, D., Hartman, K., Howell, K., and Marchand, B., "Formation Control of the MAXIM L2 Libration Orbit Mission”, AIAA/AAS Astrodynamics Specialist Conference, Providence, RI, 2004.

[4] Curtis, S., "The Magnetospheric Multiscale Mission Resolving Fundamental Processes in Space Plasmas", NASA/ TM2000-209883, NASA GSFC, Greenbelt, MD, Dec. 1999.

[5] Fridlund, C. V. M., "Darwin-The Infrared Space Interferometry Mission”, ESA Bulletin, Vol. 103, 2000, pp. 2025.

[6] Chabot, T., and Udrea, B., "XEUS Mission Guidance Navigation and Control", AIAA Guidance, Navigation, and Control Conference, Keystone, CO, 2006.

[7] Sidi, M. J., Spacecraft Dynamics and Control: A Practical Engineering Approach, Cambridge University Press, New York, NY, 1997.

[8] Vallado, D. A., and McClain, W. D., Fundamentals of Astrodynamics and Applications, Space Technology Library, Microcosm Press, El Seguendo, CA, and Kluwer Academic Publishers, Dordrecht, Netherlands, 2001.

[9] Lancaster, E. R., Blanchard, R. C., and Devaney, R. A.,
"A Note on Lambert's Theorem", Journal of Spacecraft and Rockets, Vol. 3, No. 9, 1966, pp. 1436-1438.

[10] Battin, R. H., and Vaughan, R. H., "An Elegant Lambert Algorithm", Journal of Guidance, Control, and Dynamics, Vol. 7, No. 6, 1984, pp. 662-670.

[11] Won, C-H., "Fuel- or Time-Optimal Transfers between Coplanar, Coaxial Ellipses using Lambert's Theorem", Journal of Guidance, Control, and Dynamics, Vol. 22, No. 4, 1999, pp. 536-542.

[12] Prussing, J. E., "A Class of Optimal Two-Impulse Rendezvous using Multiple-Revolution Lambert Solutions", Journal of the Astronautical Sciences, Vol. 48, No. 2-3, 2000, pp. 131-148.

[13] Shen, H., and Tsiotras, P., "Optimal Two-Impulse Rendezvous using Multiple-Revolution Lambert's Solutions", Journal of Guidance, Control, and Dynamics, Vol. 26, No. 1, 2003, pp. 50-61.

[14] Vaddi, S., Alfirend, K., Vadali, S., and Sengupta, P., "Formation Establishment and Reconfiguration using Impulsive Control", Journal of Guidance, Control, and Dynamics, Vol. 28, No. 2, 2005, pp. 262-268.

[15] Ketema, Y., “Optimal Satellite Transfers using Relative Motion Dynamics", Journal of Guidance, Control, and Dynamics, Vol. 32, No. 5, 2009, pp. 1508-1518.

[16] Schaub, H., and Junkins, J. L., Analytical Mechanics of Space Systems, AIAA Education Series, American Institute of Aeronautics and Astronautics, Reston, VA, 2003.

[17] Bae, J., and Kim, Y., "Revisiting the General Periodic Relative Motion in Elliptic Reference Orbits", Acta Astronautica, Vol. 85, 2013, pp. 100-112. 